\title{
Uremic cardiomyopathy may improve with kidney transplantation: a case report
}

\author{
Seung Hwan Song ${ }^{1}$, Geun Hong ${ }^{2}$, Ku Yong Chung ${ }^{2}$, Dami Jung ${ }^{2}$ \\ ${ }^{1}$ Division of Transplantation Surgery, Department of Surgery, Ewha Womans University Seoul Hospital, Seoul, Korea \\ ${ }^{2}$ Division of Transplantation Surgery, Department of Surgery, Ewha Womans University Mokdong Hospital, Seoul, Korea
}

Background: In patients with chronic kidney disease (CKD), left ventricular (LV) hypertrophy with impaired LV systolic function, which is called uremic cardiomyopathy is often observed. In recent studies, CKD may cause and aggravate uremic cardiomyopathy in patients without coronary artery disease. We report a case of improvement of severe uremic cardiomyopathy without coronary artery disease after kidney transplantation (KT).

Methods: A 43-year-old man, who received deceased donor KT on January 20, 2019. Time on dialysis before KT was 73 months. The cause of CKD was immunoglobulin A nephropathy. At the time of admission, an echocardiogram revealed a LV ejection fraction (LVEF) of $8 \%$, severe global hypokinesia, and an enlarged bilateral chamber. A coronary angiogram demonstrated normal coronary arteries.

Results: He underwent a deceased donor KT from marginal donor and produced urine soon after the transplantation. He was treated with the immunosuppression regimen, which included prednisone, mycophenolate mofetil, and tacrolimus. Patient was also receiving other medicines than immunosuppressants such as antihypertensive drugs, taken both before and after KT. Successful KT improved his cardiac symptoms and increased his LVEF to $16 \%$ on postoperative day 17 . His LV function improved as his LVEF increased to $32 \%$, which has been maintained along with a favorable renal allograft function for 4 months.

Conclusions: This case demonstrates the patients with severely impaired cardiac function could be able to receive significant benefits after successful KT. KT should be considered for CKD patients with LV systolic dysfunction.

Corresponding author: Seung Hwan Song

E-mail: seunghwanhappy@ewha.ac.kr

(c) The Korean Society for Transplantation

This is an Open Access article distributed under the terms of the Creative Commons Attribution Non-Commercial License (http://creativecommons.org/licenses/by-nc/4.0/) which permits unrestricted non-commercial use, distribution, and reproduction in any medium, provided the original work is properly cited. 